\title{
An exponentially fitted scheme for solving singularly perturbed delay problems
}

\author{
Rakesh Ranjan $^{\star}$ • H.S. Prasad
}

Received: 08.05.2019 / Revised: 10.01.2020 / Accepted: 02.03.2020

\begin{abstract}
This paper presents a new exponentially fitted three point scheme for solving singularly perturbed delay problems with boundary layer at left (or right) end of the domain. Scheme is derived using the exact and approximate rule of integration with finite difference approximations of first derivative. A fitting factor is introduced in the scheme using the concept of singular perturbation. Thomas algorithm is used to solve the resulting tri-diagonal system. Convergence analysis of the proposed method is given. Applicability of the method is shown by implementing it on several linear and nonlinear example problems with the various values of the delay parameter $\delta$ and the perturbation parameter $\varepsilon$. Numerical results in terms of maximum absolute errors are presented to illustrate the efficiency of the method. It is observed that the method is able to approximates the solution very well.
\end{abstract}

Keywords. singularly perturbed differential-difference equation, negative shift, boundary layer, exponential integrating factor, numerical integration

Mathematics Subject Classification (2010): 65L10, 65L11, 65L12

\section{Introduction}

It is well known that the delay differential equations arise very frequently in the mathematical modeling of various practical phenomena, such as hydrodynamics of liquid helium [9], thermo-elasticity [6], first exit time problem in the modeling of the activation function of neuronal variability [24], second-sound theory [10], reaction-diffusion equations [3], diffusion in polymers [27], a variety of model for physiological processes or diseases [28] etc. A singularly perturbed delay differential equation(SPDDE) is a differential equation in which highest derivative is multiplied with a small parameter and which contains at least one delay or advance term. In recent papers [14-18] the terms negative or left shift and positive or right shift have been used for delay and advance respectively. An useful discussion on such small delay problems can be found throughout the literature on epidemics and population where these small shifts play an important role in the modeling of various real life problems[21]. A good number of articles [12, 13, 34] and high level monographs

\footnotetext{
* Corresponding author

R. Ranjan

Department of Mathematics, National Institute of Technology, Jamshedpur

E-mail: 90.ranjan@gmail.com

H.S. Prasad

Department of Mathematics, National Institute of Technology, Jamshedpur
} 
$[2,4,5,21,8,11,29,31,32]$ are in literature which describe the various numerical and analytical methods for the solution of singular perturbation problems. Lange and Miura [22-26] suggested some asymptotic approaches in their study of a class of boundary value problems for linear second order differential-difference equations in which the highest order derivatives is multiplied by a small positive parameter. Kadalbajoo and Sharma $[14,16,19]$ considered numerical techniques by/using finite difference schemes for solving singularly perturbed delay differential-difference equations. In [7], an improved reproducing kernel method is applied for solving singularly perturbed differential-difference equation. Kadalbajoo and Kumar [17] presented B-Spline collocation method with fitted mesh technique to solve singularly perturbed differential-difference equation on uniform mesh. Kadalbajoo and Ramesh [15] proposed hybrid and midpoint upwind schemes on shishkin mesh, for capturing the behaviour of the solutions, inside and outside of the layer region respectively. Patidar and Sharma [33] presented non-standard finite difference methods to solve singularly perturbed differential-difference equations with small delay. Ramos [35] used full exponential method and partial exponential method for solving a singularly perturbed delay differential equations with small delay. In this paper we have suggested an exponentially fitted numerical integration scheme based on uniform mesh for solving singularly perturbed delay differential equations having delay in convection term which has the boundary layer at left and right end of the domain respectively. An exact and approximate rule of integration with finite difference approximations of first derivatives is used to obtain a three term recurrence relationship.

The paper is organized as follows: Statement of the problem is given in Section: 2. A brief discussion on determination of the fitting factor with left and right end boundary layer problems are presented in the subsections 2.1 and 2.2 respectively. In Section: 3, Convergence analysis of the proposed method is given. Numerical illustrations with the results in terms of maximum absolute errors are presented in tables in Section: 4. Conclusions and Discussions are presented in the last Section: 5. Paper ends with the references.

\section{Statement of the continuous problem:}

We consider the following second order singularly perturbed delay differential equations with delay in the convection term:

$$
\varepsilon y^{\prime \prime}(t)+p(t) y^{\prime}(t-\delta)+q(t) y(t)=r(t) ; 0 \leq t \leq 1
$$

subject to the interval and boundary conditions:

$$
y(t)=\eta(t),-\delta \leq t \leq 0, \text { and } y(1)=\gamma
$$

where $\varepsilon(0<\varepsilon<<1)$ is a perturbation parameter and $\gamma$ are known finite constant and $\delta=o(\varepsilon)$ is the small delay. Also $p(t), q(t), r(t)$ and $\eta(t)$ are sufficiently smooth functions. For a function $y(x)$ be a smooth solution to the problem (1)-(2), it must satisfy the boundary value problem (1)-(2), be continuous on $[0,1]$ and be continuously differentiable on $(0,1)$.

For $\delta=0$, the problem becomes an ordinary singularly perturbed problems. Depending on the nature of the coefficient functions, such problems exhibits boundary and/or interior layer behavior when singular perturbation parameter $\varepsilon$ tends to zero. The solution of the boundary value problem (1) with (2) exhibits layer behavior at the left or right end points of the interval depending on whether $p(t)>0$ or $p(t)<0$ on $[0,1]$.

In this paper, both the cases, i.e., when the solution exhibits boundary layer behavior on the left side and on the right side are studied.

We assume that $p(t) \geq K>0$ and $q(t)<0$ for $\forall t \in[0,1]$. Under these assumptions the above problem (1)with (2) has unique solution which exhibits a boundary layer at $t=0$. 
Since, here we assume that the delay parameter is smaller than the perturbation parameter (i.e., $\delta<\varepsilon$ ), approximating $y^{\prime}(t-\delta)$ by the linear interpolation, we have

$$
y^{\prime}(t-\delta) \approx y^{\prime}(t)-\delta y^{\prime \prime}(t)
$$

Substituting (3) in to equation (1), we get

$$
\begin{aligned}
& L_{\tau} y(t)=(\varepsilon-\delta p(t)) y^{\prime \prime}(t)+p(t) y^{\prime}(t)+q(t) y(t)=r(t) ; 0 \leq t \leq 1 \\
& L_{\tau} y(t)=\mu y^{\prime \prime}(t)+p(t) y^{\prime}(t)+q(t) y(t)=r(t) ; 0 \leq t \leq 1 \\
& L_{\tau} y(t)=\varepsilon y^{\prime \prime}(t)+a(t) y^{\prime}(t)+b(t) y(t)=f(t) ; 0 \leq t \leq 1
\end{aligned}
$$

with the boundary condition $y(0)=\eta(0)=\eta_{0}$ (say), $y(1)=\gamma$

where $a(t)=\frac{\varepsilon p(t)}{\varepsilon-\delta p(t)}, b(t)=\frac{\varepsilon q(t)}{\varepsilon-\delta p(t)}, f(t)=\frac{\varepsilon r(t)}{\varepsilon-\delta p(t)}$

Here we assume that $\mu=(\varepsilon-\delta p(t))>0$, then we have $a(t) \geq a^{*}>0$ and $b(t) \geq-b^{*}<0$ for some positive constants $a^{*}, b^{*}$.

For appropriate choices of $\delta$ such that $0 \leq \mu=\varepsilon-\delta \zeta<<1$, where $\zeta=\min _{0 \leq t \leq 1} p(t)$.

The operator $L_{\tau}=\varepsilon \frac{d^{2}}{d t^{2}}+a(t) \frac{d}{d t}+b(t) I$ in (4) satisfies the following minimum principle.

Lemma 2.1 Suppose $\psi(t)$ is a smooth function satisfying $\psi(0) \geq 0, \psi(1) \geq 0$. Then $L_{\tau} \psi(t) \leq 0, \forall t \in(0,1)$ implies $\psi(t) \geq 0, \forall t \in[0,1]$.

Proof. Let $k \in[0,1]$ be such that $\psi(k)<0$ and $\psi(k)=\min _{x \in[0,1]} \psi(x)$. Clearly $k \notin\{0,1\}$, therefore $\psi^{\prime}(k)=0$ and $\psi^{\prime \prime}(k) \geq 0$. Therefore, we obtain

$$
\begin{aligned}
L_{\tau} \psi(k) & =(\varepsilon-\delta p(x)) \psi^{\prime \prime}(k)+p(k) \psi^{\prime}(k)+p(k) \psi(k)>0, \\
\text { or }, L_{\tau} \psi(k) & =\varepsilon \psi^{\prime \prime}(k)+a(k) \psi^{\prime}(k)+b(k) \psi(k)>0,
\end{aligned}
$$

which is a contradictory to our assumption. Hence it is proved that $\psi(k) \geq 0$ and thus $\psi(t) \geq 0 \forall t \in[0,1]$.

Lemma 2.2 Let $y(t)$ be the solution of the problem (4)and (5) then we have

$$
\|y\| \leq \theta^{-1}\|f\|+\max \left(\left|\eta_{0}\right|,|\gamma|\right) .
$$

where $\|\cdot\|$ is the $L_{\infty}$ norm given by $\|y\|=\max _{0 \leq t \leq 1}|y(t)|$.

Proof. Let $\psi^{ \pm}(t)$ be two barrier functions define by

Then this implies,

$$
\psi^{ \pm}(t)=\theta^{-1}\|f\|+\max \left(\left|\eta_{0}\right|,|\gamma|\right) \pm y(t)
$$

$$
\begin{aligned}
& \begin{aligned}
\psi^{ \pm}(0) & =\theta^{-1}\|f\|+\max \left(\left|\eta_{0}\right|,|\gamma|\right) \pm y(0) \\
& =\theta^{-1}\|f\|+\max \left(\left|\eta_{0}\right|,|\gamma|\right) \pm \eta_{0} \text { since } y(0)=\eta(0)=\eta_{0} \\
& \geq 0
\end{aligned} \\
& \begin{aligned}
\psi^{ \pm}(1) & =\theta^{-1}\|f\|+\max \left(\left|\eta_{0}\right|,|\gamma|\right) \pm y(1) \\
& =\theta^{-1}\|f\|+\max \left(\left|\eta_{0}\right|,|\gamma|\right) \pm \gamma \text { since } y(1)=\gamma \\
\Rightarrow L_{\tau} \psi^{ \pm}(t) & =\varepsilon\left(\psi^{ \pm}(t)\right)^{\prime \prime}+a(t)\left(\psi^{ \pm}(t)\right)^{\prime}+b(t) \psi^{ \pm}(t) \\
& =b(t)\left[\theta^{-1}\|f\|+\max \left(\left|\eta_{0}\right|,|\gamma|\right)\right] \pm L_{\tau} y(t) \\
& =b(t)\left[\theta^{-1}\|f\|+\max \left(\left|\eta_{0}\right|,|\gamma|\right)\right] \pm f(t) \text { using }(2.4)
\end{aligned}
\end{aligned}
$$

As $b(t) \leq-\theta<0$ implies $b(t) \theta^{-1} \leq-1$ and since $\|f\| \geq f(t)$, we have

$$
\Rightarrow L_{\tau} \psi^{ \pm}(t) \leq(-\|f\| \pm f(t))+b(t) \max \left(\left|\eta_{0}\right|,|\gamma|\right) \leq 0, \forall t \in[0,1] .
$$

Thus by the minimum principle we obtain, $\psi^{ \pm} \geq 0 \forall t \in[0,1]$. Now in order to compute the error in our numerical integration approximations we shall require a bounded for the derivative of the solution $y(t)$ that is valid for all $\tau \in(0,1]$. 
By using Lemma 1, we obtain the required estimate.

Theorem 2.1 Let $y(t)$ be the solution of the Problem (4) and (5), then independent of $\varepsilon$. $\left\|y^{(m)}\right\| \leq C(\varepsilon-\delta K)^{-m}$ for $m=1,2,3$. where $C$ is a positive constant

Proof. Let $t \in(0,1)$ and let $V_{t}=(c, c+\beta)$ be a neighborhood of $t$, where $\beta>$ E i.e. $\beta=$ $\varepsilon-\delta\|p\|$ is a constant and also $c$ being a positive constant chosen so that $t \in V_{t}$ and $V_{t} \subset(0,1)$, then by mean value theorem there exist a point $\xi \in V_{t}$ such that

so

$$
y^{\prime}(\xi)=\frac{y(c+(\varepsilon-\delta K))-y(c)}{\varepsilon-\delta K}
$$

$$
(\varepsilon-\delta K)\left\|y^{\prime}(\xi)\right\| \leq 2\|y\|
$$

Now differentiating (4) from $\xi$ to $t$ and taking the modulus on both the sides and by using the fact that the maximum norm of a function is always greater than the value of the function over the domain, we obtain

$$
(\varepsilon-\delta K)\left|y^{\prime}(t)\right| \leq(\varepsilon-\delta K)\left|y^{\prime}(\xi)\right|+\|f\||t-\xi|+\int_{\xi}^{t}\left|a(x) y^{\prime}(x)\right| d x+\|b\|\|y\||t-\xi| .
$$

we known that

$$
\int_{\xi}^{t}\left|a(x) y^{\prime}(x)\right| d x \leq\left(2\|a\|+\left\|a^{\prime}\right\||t-\xi|\right)\|y\|,
$$

with inequalities (8), (10) and using the fact $|t-\xi|<\beta$ and Lemma 2, from (9) we obtain $\left\|y^{\prime}(t)\right\| \leq C(\varepsilon-\delta K)^{-1}$

where $C=\|f\|+\left(2+2\|a\|+\left\|a^{\prime}\right\|+\|b\|\right)\left(\theta^{-1}\|f\|+\max \left(\left|\eta_{0}\right|,|\gamma|\right)\right)$ which is a positive independent of $\varepsilon$. The bounds for $\left\|y^{\prime \prime}\right\|$ and $\left\|y^{\prime \prime \prime}\right\|$ can be obtained similarly by Miller and O'Riordan [30] using the techniques given by Kellogg and Tsan [20].

In order to prove that the numerical method is $\varepsilon$-uniform, one needs more information about the exact solution. Let us decompose the solution $y$ into a smooth $(v)$ and a singular (z) component as follows:-

$$
y=v+z,
$$

where $v$ and $z$ are smooth and singular components respectively. The smooth component $z$ can be written in three term asymptotic expansion as $v(t)=v_{0}(t)+(\varepsilon-\delta K) v_{1}(t)+$ $(\varepsilon-\delta K)^{2} v_{2}(x)$ and $v_{0}, v_{1}$ and $v_{2}$ and defined to be the solution of the problems as follows:-

$$
\begin{aligned}
& a(t) v^{\prime}{ }_{0}(t)+b(t) v_{0}(t)=f(t), t \in(0,1), v_{0}(1)=y(1), \\
& a(t) v_{1}^{\prime}(t)+b(t) v_{1}(t)=-(\varepsilon-\delta p(t)) v^{\prime \prime}{ }_{0}(t) /(\varepsilon-\delta K) ; t \in(0,1), v_{0}(1)=0, \\
& L_{\tau} v_{2}(t)=-(\varepsilon-\delta p(t)) v^{\prime \prime}{ }_{1}(t) /(\varepsilon-\delta K), t \in(0,1), v_{2}(0)=0, v_{2}(1)=0 .
\end{aligned}
$$

The smooth component $v$ is the solution of

$$
L_{\tau} v(t)=f(t), t \in(0,1), v(0)=v_{0}(0)+(\varepsilon-\delta K) v_{1}(0), v(1)=y(1)
$$

and the singular component $z$ is the solution of the homogeneous problem

$$
L_{\tau} z(t)=0, t \in(0,1), z(0)=y(0)-v(0), z(1)=0 .
$$

Now we can state the following theorem on the bounds for the solutions and derivatives of (11) and (12). 
Theorem 2.2 Let $y$ be the solution of (4) and (5) and let $y=v+z$. For $0 \leq m \leq 3$ and for sufficiently small $\varepsilon, v, z$ and their derivatives satisfy the following bounds:

$$
\begin{gathered}
\left\|v^{(m)}\right\| \leq C(\varepsilon-\delta K)^{2-m}, \\
|z(t)| \leq C \exp (-K t /(\varepsilon-\delta K)), t \in(0,1), \\
\left\|u^{(m)}\right\| \leq C(\varepsilon-\delta K)^{-m} \exp (-K t /(\varepsilon-\delta K)), t \in(0,1) .
\end{gathered}
$$

Proof. For the proof of the above theorem can be found in [14].

\subsection{Description of the method for left-End Boundary Layer Problems:}

The solutions of (4) with (5) are known to be of the following form (cf.[32], pp.22-26):

$$
y(t)=y_{0}(t)+\frac{p(0)}{p(t)}\left(\eta_{0}-y_{0}(0)\right) e^{-\int_{0}^{t}\left(\frac{p(t)}{\mu}-\frac{q(t)}{p(t)}\right) d t}+o(\varepsilon)
$$

where $y_{0}(t)$ denotes the solution of the following problem:

$$
p(t) y_{0}^{\prime}(t)+q(t) y_{0}(t)=r(t) ; u_{0}(1)=\gamma
$$

Under the consideration of Taylor's series expansions for $p(t)$ and $q(t)$ about the point ' $t=0^{\prime}$ upto their first terms only, the equation (16) becomes:

$$
y(t)=y_{0}(t)+\left(\eta_{0}-y_{0}(0)\right) e^{-\left(\frac{p(0)}{\mu}-\frac{q(0)}{p(0)}\right) t}+o(\varepsilon)
$$

Further, considering equation (18) at the point $t=t_{i}=i h, i=0,1,2, \ldots, N$ and taking the limit as $h \rightarrow 0$ we obtain

$$
\lim _{h \rightarrow 0} y(i h)=y_{0}(0)+\left(\eta_{0}-y_{0}(0)\right) e^{-\left(\frac{a^{2}(0)-\mu b(0)}{a(0)}\right) i \rho}+o(\varepsilon)
$$

where $\rho=h / \mu$.

Now, writing the equation (4) in the following form:

$$
\mu y^{\prime \prime}+[A(t)]^{\prime}+B(t) y(t)=C(t) ; \quad 0 \leq x \leq 1
$$

where, $A(t)=p(t) y(t), B(t)=q(t)-p^{\prime}(t)$ and $C(t)=r(t)$ and using the usual rule of exact and trapezoidal rule of integration over $\left[t_{i}, t_{i+1}\right](i=1,2, \ldots, N-1)$ the equation (20) becomes:

$$
\mu y_{i+1}^{\prime}-\mu y_{i}^{\prime}+p_{i+1} y_{i+1}-p_{i} y_{i}+\frac{h}{2}\left[B_{i+1} y_{i+1}+B_{i} y_{i}\right]=\frac{h}{2}\left[C_{i+1}+C_{i}\right]
$$

Now approximating the first order derivative $y_{i+1}^{\prime}$ using non-symmetric finite difference analogue:

$$
y_{i+1}^{\prime}=\frac{y_{i-1}-4 y_{i}+3 y_{i+1}}{2 h}+o\left(h^{2}\right)
$$

and $y_{i}^{\prime}$ by mixed finite difference analogue(a combination of central and upwind finite difference approximations):

i.e.,

$$
y_{i}^{\prime}=\alpha\left(\frac{y_{i+1}-y_{i-1}}{2 h}\right)+(1-\alpha)\left(\frac{y_{i+1}-y_{i}}{h}\right)+o(h) \text { with } \alpha=\frac{1}{2}
$$

$$
y_{i}^{\prime} \approx \frac{3 y_{i+1}-2 y_{i}-y_{i-1}}{4 h}+o(h)
$$


with notations: $y\left(t_{i}\right)=y_{i}, y\left(t_{i+1}\right)=y_{i+1}, B\left(t_{i}\right)=B_{i}, C\left(t_{i}\right)=C_{i}, p\left(t_{i}\right)=p_{i}$ etc. in (21), we get:

$$
\begin{aligned}
\frac{\mu}{2 h}\left(y_{i-1}-4 y_{i}+3 y_{i+1}\right)-\frac{\mu}{3 h}\left(2 y_{i+1}-y_{i}-y_{i-1}\right)-\left(p_{i} y_{i}-p_{i+1} y_{i+1}\right) \\
+\frac{h}{2}\left[B_{i+1} y_{i+1}+B_{i} y_{i}\right]=\frac{h}{2}\left[C_{i+1}+C_{i}\right] ; 0 \leq i \leq N-1
\end{aligned}
$$

Now, we introduce a fitting factor $\sigma(\rho)$ in the above scheme (22)as,

$$
\begin{gathered}
\frac{\sigma(\rho) 3 \mu}{4 h}\left(y_{i-1}-2 y_{i}+y_{i+1}\right)-\left(p_{i} y_{i}-p_{i+1} y_{i+1}\right)+\frac{h}{2}\left[B_{i+1} y_{i+1}+B_{i} y_{i}\right] \\
=\frac{h}{2}\left[C_{i+1}+C_{i}\right] ; 0 \leq i \leq N-1
\end{gathered}
$$

with $y(0)=\eta$ and $y(1)=\gamma$.

The fitting factor $\sigma(\rho)$ is to be determined in such a way that the solution of (23) converges uniformly to the solution of (4)-(5). In the limit as $h \rightarrow 0$ the equation (23) becomes:

$$
\begin{array}{r}
\lim _{h \rightarrow 0}\left[\frac{3 \sigma(\rho)}{4 \rho}(y(i h-h)-2 y(i h)+y(i h+h))\right]-\lim _{h \rightarrow 0}[p(i h) y(i h) \\
-p(i h+h) y(i h+h)]=0
\end{array}
$$

under the assumption that $\frac{1}{2}\left[B_{i+1} y_{i+1}+B_{i} y_{i}\right]$ and $\frac{1}{2}\left[C_{i+1}+C_{i}\right]$ are bounded. Substituting equation (19) in equation (24) and simplifying, we get a constant fitting factor

$$
\sigma(\rho)=\frac{4 p(0) \rho}{6}\left[\frac{e^{-\left(\frac{p^{2}(0)-\mu q(0)}{p(0)}\right) \frac{\rho}{2}}}{\sinh \left(\left(\frac{p^{2}(0)-\mu q(0)}{p(0)}\right) \frac{\rho}{2}\right)}\right]
$$

Rearranging the equation (23) we get a scheme as a three term recurrence relationship of the form:

$$
E_{i} y_{i-1}-F_{i} y_{i}+G_{i} y_{i+1}=H_{i}, \quad(i=1,2,3, \ldots, N-1)
$$

where

$$
\begin{aligned}
& E_{i}=\frac{3 \sigma \mu}{4 h} \\
& F_{i}=\frac{6 \sigma \mu}{4 h}+p_{i}-\frac{h}{2} B_{i} \\
& G_{i}=\frac{3 \sigma \mu}{4 h}+p_{i+1}+\frac{h}{2} B_{i+1} \\
& H_{i}=\frac{h}{2}\left[C_{i}+C_{i+1}\right]
\end{aligned}
$$

Equation (26) gives a system of $(N-1)$ equations with $(N-1)$ unknowns $y_{1}$ to $y_{N-1}$. These $(N-1)$ equations together with the Eq. (5) are sufficient to solve the tri-diagonal system by using Thomas Algorithm also called "Discrete Invariant Imbedding algorithm". The conditions for the discrete invariant embedding algorithm to be stable are : $E_{i}>$ $0, F_{i}>0, F_{i} \geq E_{i}+G_{i}$ provided $p(x)$ is decreasing function and $\left(B_{i}+B_{i+1}\right) \leq 0$ and $\left|E_{i}\right| \leq\left|G_{i}\right|$; provided $\left(p_{i+1}+\frac{h}{2} B_{i+1}\right) \geq 0$ In this method, if the assumptions $a(x)>$ $0, b(x)<0$ and $(\varepsilon-\delta a(x))>0$ hold, one can easily show that the conditions given in (24) hold and thus the invariant imbedding algorithm is stable.

Thus the coefficient matrix of the tri-diagonal system of equations (26) with boundary conditions (5) is diagonally dominant and hence non-singular.

\subsection{Description of the method for Right-End Boundary Layer Problems:}

We now assume that $p(t) \leq K<0$ throughout the interval $[0,1]$, where $K$ is some negative constant. This assumption merely implies that the boundary layer for equation (4)(5) will be in the neighborhood of $t=1$. From the theory of singular perturbation, it is known that the solution of (4) with (5) is of the from [cf. O Malley[32]; pp. 22-26]:

$$
y(t)=y_{0}(t)+\frac{p(1)}{p(t)}\left(\gamma-y_{0}(1)\right) e^{\int_{t}^{1}\left(\frac{p(t)}{\mu}-\frac{q(t)}{p(t)}\right) d t}+o(\varepsilon)
$$


where $y_{0}(t)$ represents the solution of the reduced problem:

$$
p(t) y_{0}^{\prime}(t)+q(t) y_{0}(t)=r(t) ; y_{0}(0)=\eta \text {. }
$$

For appropriate choices of $\delta$ such that $0 \leq \mu=\varepsilon-\delta \omega<<1$, where $\omega=\max _{0 \leq t \leq 1} p(t)$. By expanding $p(t)$ and $q(t)$ in (27) with the help of the Taylor's series about the point ' $t=1^{\prime}$ and restricting to their first terms, we obtain:

$$
y(t)=y_{0}(t)+\left(\gamma-y_{0}(1)\right) e^{\left(\frac{p(1)}{\mu}-\frac{q(1)}{p(1)}\right)(1-t)}+o(\varepsilon)
$$

Further, considering equation(28) at the point $t=t_{i}=i h, i=0,1,2, \ldots, N$ and taking the limit as $h \rightarrow 0$ we obtain

$$
\lim _{h \rightarrow 0} y(i h)=y_{0}(0)+\left(\gamma-y_{0}(1)\right) e^{\left(\frac{p^{2}(1)-\mu q(1)}{p(1)}\right)\left(\frac{1}{\mu}-i \rho\right)}+o(\varepsilon)
$$

where $\rho=h / \mu$. Integrating equation (20) in $\left[t_{i-1}, t_{i}\right]$ using the exact and trapezoidal rule of integration, we Obtain

$$
\mu y_{i}^{\prime}-\mu y_{i-1}^{\prime}+p_{i} y_{i}-p_{i-1} y_{i-1}+\frac{h}{2}\left[B_{i} y_{i}+B_{i-1} y_{i-1}\right]=\frac{h}{2}\left[C_{i}+C_{i-1}\right]
$$

Now approximating the first order derivative $y_{i-1}^{\prime}$ using non-symmetric finite difference analogue:

$$
y_{i-1}^{\prime}=\frac{-3 y_{i-1}+4 y_{i}-y_{i+1}}{2 h}+o\left(h^{2}\right)
$$

and $y_{i}^{\prime}$ by mixed finite difference analogue(a combination of central and upwind finite difference approximations):

i.e.,

$$
y_{i}^{\prime}=\alpha\left(\frac{y_{i+1}-y_{i-1}}{2 h}\right)+(1-\alpha)\left(\frac{y_{i+1}-y_{i}}{h}\right)+o(h) \text { with } \alpha=\frac{1}{2}
$$

$$
y_{i}^{\prime} \approx \frac{3 y_{i+1}-2 y_{i}-y_{i-1}}{4 h}+o(h)
$$

with notations: $y\left(t_{i}\right)=y_{i}, y\left(t_{i+1}\right)=y_{i+1}, B\left(t_{i}\right)=B_{i}, C\left(t_{i}\right)=C_{i}, p\left(t_{i}\right)=p_{i}$ etc. in (30), we obtain the following scheme:

$$
\begin{gathered}
\mu\left(\frac{3 y_{i+1}-2 y_{i}-y_{i-1}}{4 h}\right)-\mu\left(\frac{-3 y_{i-1}+4 y_{i}-y_{i+1}}{2 h}\right)+p_{i} y_{i}-p_{i-1} y_{i-1} \\
+\frac{h}{2}\left[B_{i} y_{i}+B_{i-1} y_{i-1}\right]=\frac{h}{2}\left[C_{i}+C_{i-1}\right] ; 0 \leq i \leq N-1
\end{gathered}
$$

An introduction of a fitting factor $\sigma(\rho)$ in the above scheme (31) gives:

$$
\begin{gathered}
\frac{\sigma(\rho) 5 \mu}{4 h}\left(y_{i-1}-2 y_{i}+y_{i+1}\right)-\left(p_{i-1} y_{i-1}-p_{i} y_{i}\right)+\frac{h}{2}\left[B_{i} y_{i}+B_{i-1} y_{i-1}\right] \\
=\frac{h}{2}\left[C_{i}+C_{i-1}\right] ; 0 \leq i \leq N-1
\end{gathered}
$$

with $y(0)=\eta$ and $y(1)=\gamma$.

The fitting factor $\sigma(\rho)$ is to be determined in such a way that the solution of (32) converges uniformly to the solution of (4)-(5).

In the limit as $h \rightarrow 0$ the equation (32) becomes:

$$
\begin{gathered}
\lim _{h \rightarrow 0}\left[\frac{5 \sigma(\rho)}{4 \rho}(y(i h-h)-2 y(i h)+y(i h+h))\right]-\lim _{h \rightarrow 0}[p(i h-h) y(i h-h) \\
-p(i h) y(i h)]=0
\end{gathered}
$$

under the assumption that $\frac{1}{2}\left[B_{i} y_{i}+B_{i-1} y_{i-1}\right]$ and $\frac{1}{2}\left[C_{i}+C_{i-1}\right]$ are bounded. Substituting equation (29) in equation (33) and simplifying, we get a constant fitting factor

$$
\sigma(\rho)=\frac{4 p(0) \rho}{10}\left[\frac{e^{\left(\frac{p^{2}(0)-\mu q(0)}{p(0)}\right) \frac{\rho}{2}}}{\sinh \left(\left(\frac{p^{2}(0)-\mu q(0)}{p(0)}\right) \frac{\rho}{2}\right)}\right]
$$


Rearranging the equation (32) we get a scheme as a three term recurrence relationship of the form:

$$
E_{i} y_{i-1}-F_{i} y_{i}+G_{i} y_{i+1}=H_{i},(i=1,2,3, \ldots, N-1)
$$

where

$$
\begin{aligned}
& E_{i}=\frac{5 \sigma \mu}{4 h}-p_{i-1}+\frac{h}{2} B_{i-1} \\
& F_{i}=\frac{10 \sigma \mu}{4 h}-p_{i}-\frac{h}{2} B_{i} \\
& G_{i}=\frac{5 \sigma \mu}{4 h} \\
& H_{i}=\frac{h}{2}\left[C_{i}+C_{i-1}\right]
\end{aligned}
$$

Equation (35) gives a system of $(N-1)$ equations with $(N-1)$ unknowns $y_{1}$ to $y_{N-1}$. These $(N-1)$ equations together with the equation (5) are sufficient to solve the tridiagonal system by using Thomas Algorithm also called 'Discrete Invariant Imbedding algorithm'. The conditions for the discrete invariant embedding algorithm to be stable are : $E_{i}>0, F_{i}>0, F_{i} \geq E_{i}+G_{i}$; provide $p(x)$ is decreasing function and $\left(B_{i-1}+B_{i}\right) \leq 0$. and

$$
\left|E_{i}\right| \leq\left|G_{i}\right| ; \text { Provide }\left(-p_{i-1}+\frac{h}{2} B_{i}\right) \leq 0
$$

In this method, if the assumptions $a(x)>0, b(x)<0$ and $(\varepsilon-\delta a(x))>0$ hold, one can easily show that the conditions given in (35) hold and thus the invariant embedding algorithm is stable.

\section{Convergence analysis:}

In this section we will discuss the error analysis of the proposed method and show that the method is of first order accurate on uniform mesh. Writing the tridiagonal system (26) in matrix-vector form [1]:, we get

$$
D Y=M
$$

where $D=\left(u_{i, j}\right), 1 \leq i, j \leq N-1$ is a tri-diagonal matrix of order N-1, with

$$
\begin{aligned}
u_{i, i+1} & =-\left(\frac{3 \sigma \mu}{4}+h p_{i+1}+\frac{h^{2}}{2} B_{i+1}\right) \\
u_{i, i} & =\frac{6 \sigma \mu}{4}+h p_{i}-\frac{h^{2}}{2} B_{i} \\
u_{i, i-1} & =\frac{-3 \sigma \mu}{4}
\end{aligned}
$$

where $M=\left(g_{i}\right)$ is a column vector with $g_{i}=-\frac{h^{2}}{2}\left[C_{i}=C_{i+1}\right], i=1,2, \ldots, N-1$ with local truncation error

$$
\tau_{i}(h)=h^{2}\left[p_{i} y_{i}+p_{i}^{\prime} y_{i}^{\prime}+\frac{3 \mu}{4} y_{i}^{\prime \prime}-C_{i}\right]+o\left(h^{3}\right)
$$

We also have

$$
D \bar{Y}-\tau(h)=M
$$

where $\bar{Y}=\left(\bar{Y}_{0}, \bar{Y}_{1}, \bar{Y}_{2}, \ldots, \bar{Y}_{N}\right)^{T}$ denotes the actual solution and $\tau(h)=\left(\tau_{1}(h), \tau_{2}(h), \ldots, \tau_{N}(h)\right)^{T}$ is the local truncation error.

From the equations (37) and (39), we get

$$
D(\bar{Y}-Y)=\tau(h)
$$

Thus, we obtain the error equation

$$
D E=\tau(h)
$$


where $E=\bar{Y}-Y=\left(e_{0}, e_{1}, e_{2}, \ldots, e_{N}\right)^{T}$.

Let $S_{i}$ be the sum of elements of the $i^{\text {th }}$ row of $\mathrm{D}$, then we have

$$
\begin{aligned}
& S_{1}=\sum_{j=1}^{N-1} u_{1, j}=\frac{3 \sigma \mu}{4}+h\left[p_{1}-p_{2}\right]-\frac{h^{2}}{2}\left[B_{1}+B_{2}\right] \text { for } i=1 \\
& S_{i}=\sum_{j=1}^{N-1} u_{i, j}=h\left[p_{i}-p_{i+1}\right]-\frac{h^{2}}{2}\left[B_{i}+B_{i+1}\right] ; \text { for } i=2(1) N-2 . \\
& S_{N-1}=\sum_{j=1}^{N-1} u_{N-1, j}=\frac{3 \sigma \mu}{4}+h p_{N-1}-\frac{h^{2}}{2} B_{N-1} \text { for } i=N-1
\end{aligned}
$$

Since $0<\epsilon<<1$, for a given $\mathrm{h}$, the matrix $\mathrm{D}$ is irreducible and monotone. Hence $D^{-1}$ exists and $D^{-1} \geq 0$.

From the error equation (41), we have

$$
E=D^{-1} \tau(h)
$$

and

$$
\|E\| \leq\left\|D^{-1}|\||| \tau(h)\|\right.
$$

Let $\bar{u}_{k, i}$ be the $(k, i)^{t h}$ elements of $D^{-1}$. Since $\bar{u}_{k, i} \geq 0$, from the theory of matrices we have

$$
\sum_{i=1}^{N-1} \bar{u}_{k, i} S_{i}=1 ; \quad k=1,2, \ldots, N-1
$$

Therefore, it follows that

for some $i$ between 1 and $\mathrm{N}-1$.

$$
\sum_{i=1}^{N-1} \bar{u}_{k, i} \leq \frac{1}{\min _{0 \leq i \leq N-1} S_{i}}=\frac{1}{h\left|p_{i}-p_{i+1}\right|}
$$

Now, we define

and $\|\tau(h)\|=\min _{0 \leq k \leq N-1}|\tau(h)|$.

$$
\left\|D^{-1}\right\|=\max _{0 \leq k \leq N-1} \sum_{i=1}^{N-1}\left|\bar{u}_{k, i}\right|
$$

Therefore, from equations (38),(42)and (43) we obtain

Which implies

$$
e_{j}=\sum_{i=1}^{N-1} \bar{u}_{k, i} \tau_{i}(h) ; j=1(1) N-1
$$

$$
\left\|e_{j}\right\| \leq \frac{k h^{2}}{h\left|p_{i}-p_{i+1}\right|} ; j=1(1) N-1
$$

where $k=p_{i}\left|y_{i}\right|+p_{i}^{\prime}\left|y_{i}^{\prime}\right|+\frac{3 \mu}{4}\left|y_{i}^{\prime \prime}\right|-C_{i}$ is constant independent of $\mathrm{h}$.

Therefore, using the definitions and equation (44), we obtain:

$$
\|E\|=o(h)
$$

Thus the method proposed is a first order convergent method on uniform mesh for left end layer problems.

As above, we can show that the scheme: (35) derived for the solution of right layer problems is of first order convergence on uniform mesh.

\section{Algorithm of the method and Numerical Illustrations:}

To solve problems of the form given in equation (1)-(2), we have followed the following algorithm:

Step 1. Modify the Singularly Perturbed Delay Differential Equation (1) to the form (4).

Step 2. Introduce the fitting parameter in (4) and determine its value using the theory of 
singular perturbation.

Step 3. Solve the tri-diagonal system (26) for the left layer problems and (35)for the right layer problems with the boundary conditions (5) using Thomas Algorithm by taking different values of the perturbation and the delay parameter.

We have implemented the above algorithm on five standard linear/nonlinear example problems having boundary layer at left/right end of the underlying interval for different values of delay parameter $\delta$ and perturbation parameter $\varepsilon$. These problems are chosen due to their wide discussion in the literature. The exact solutions for these problems are not known. Numerical solution in terms of the maximum absolute errors are computed using double - mesh principles given by

$$
Z_{N}=\min _{1 \leq i \leq N-1}\left|y_{i}^{N}-y_{2 i}^{2 N}\right|, i=1,2, \ldots, N-1
$$

Where $y_{i}^{N}$ is the numerical solution obtained on the mesh $\left\{x_{i}\right\}_{1}^{N-1}$ at the nodal point $x_{i}$ and $x_{i}=x_{0}+i h, i=1,2, \ldots, N-1$ and $y_{2 i}^{2 N}$ is the numerical solution on a mesh, obtained by halving of the original mesh size $h$ with $2 N$ number of mesh intervals.

Example 1 First we consider the singularly perturbed convection delay problem with variable coefficient exhibiting left-end boundary layer

$$
\varepsilon y^{\prime \prime}(x)+e^{-0.5 x} y^{\prime}(x-\delta)-y(x)=0 ; x \in[0,1]
$$

subject to the interval and boundary conditions

$$
y(x)=1, \quad-\delta \leq x \leq 0 \text { and } y(1)=1
$$

The exact solution is not known for this problem. The absolute maximum errors are given in Tables $\mathbf{1}, \mathbf{2}$ for $\delta=0.1 * \varepsilon$ and $\delta=0.5 * \varepsilon$ respectively.

The computational results(Absolute Maximum Error)presented in Table-1, 2 for example problem-1 show that the present scheme is capable of producing uniformly convergent solution in case when $\epsilon$ tends to zero for any fixed value of the step size $h=1 / N$.

Table 1 Computational results(MAE) for various values of $\epsilon$ and $N$ for example problem-1 with $\delta=0.1 * \varepsilon$.

\begin{tabular}{|c|cccccc|}
\hline$\epsilon$ & $N=8$ & $N=16$ & $N=32$ & $N=64$ & $N=128$ & $N=256$ \\
\hline $10^{-2}$ & $8.56280 \mathrm{E}-04$ & $1.09428 \mathrm{E}-03$ & $2.33239 \mathrm{E}-03$ & $2.26283 \mathrm{E}-03$ & $1.48615 \mathrm{E}-03$ & $8.34912 \mathrm{E}-04$ \\
$10^{-4}$ & $8.02338 \mathrm{E}-04$ & $1.99616 \mathrm{E}-04$ & $4.99785 \mathrm{E}-05$ & $1.29044 \mathrm{E}-05$ & $3.15905 \mathrm{E}-06$ & $1.07288 \mathrm{E}-06$ \\
$10^{-6}$ & $8.02338 \mathrm{E}-04$ & $1.99616 \mathrm{E}-04$ & $4.99785 \mathrm{E}-05$ & $1.29044 \mathrm{E}-05$ & $3.15905 \mathrm{E}-06$ & $9.53674 \mathrm{E}-07$ \\
$10^{-8}$ & $8.02338 \mathrm{E}-04$ & $1.99616 \mathrm{E}-04$ & $4.99785 \mathrm{E}-05$ & $1.29044 \mathrm{E}-05$ & $3.15905 \mathrm{E}-06$ & $9.53674 \mathrm{E}-07$ \\
$10^{-10}$ & $8.02338 \mathrm{E}-04$ & $1.99616 \mathrm{E}-04$ & $4.99785 \mathrm{E}-05$ & $1.29044 \mathrm{E}-05$ & $3.15905 \mathrm{E}-06$ & $9.53674 \mathrm{E}-07$ \\
$10^{-15}$ & $8.02338 \mathrm{E}-04$ & $1.99616 \mathrm{E}-04$ & $4.99785 \mathrm{E}-05$ & $1.29044 \mathrm{E}-05$ & $3.15905 \mathrm{E}-06$ & $9.53674 \mathrm{E}-07$ \\
$10^{-20}$ & $8.02338 \mathrm{E}-04$ & $1.99616 \mathrm{E}-04$ & $4.99785 \mathrm{E}-05$ & $1.29044 \mathrm{E}-05$ & $3.15905 \mathrm{E}-06$ & $9.53674 \mathrm{E}-07$ \\
$10^{-30}$ & $8.02338 \mathrm{E}-04$ & $1.99616 \mathrm{E}-04$ & $4.99785 \mathrm{E}-05$ & $1.29044 \mathrm{E}-05$ & $3.15905 \mathrm{E}-06$ & $9.53674 \mathrm{E}-07$ \\
\hline
\end{tabular}

Example 2 We consider the singularly perturbed convection delay nonlinear problem with constant coefficient exhibiting left-end boundary layer

$$
\varepsilon y^{\prime \prime}(x)+y(x) y^{\prime}(x-\delta)-y(x)=0 ; x \in[0,1]
$$

subject to the interval and boundary conditions

The linear form is:

$$
y(x)=1,-\delta \leq x \leq 0, y(1)=1
$$

$$
\varepsilon y^{\prime \prime}(x)+y^{\prime}(x-\delta)-y(x)=0 ; x \in[0,1] \text { with } y(0)=1 \text { and } y(1)=1
$$

The exact solution is not known for this problem. The absolute maximum errors are given in Tables 3, 4 for $\delta=0.1 * \varepsilon$ and $\delta=0.5 * \varepsilon$ respectively. 
Table 2 Computational results(MAE) for various values of $\epsilon$ and $N$ for example problem-1 with $\delta=0.5 * \varepsilon$.

\begin{tabular}{|c|cccccc|}
\hline$\epsilon$ & $N=8$ & $N=16$ & $N=32$ & $N=64$ & $N=128$ & $N=256$ \\
\hline $10^{-2}$ & $8.02726 \mathrm{E}-04$ & $2.56807 \mathrm{E}-04$ & $6.98358 \mathrm{E}-04$ & $1.35913 \mathrm{E}-03$ & $1.22151 \mathrm{E}-03$ & $7.77215 \mathrm{E}-04$ \\
$10^{-4}$ & $8.02338 \mathrm{E}-04$ & $1.99616 \mathrm{E}-04$ & $4.99785 \mathrm{E}-05$ & $1.29044 \mathrm{E}-05$ & $3.15905 \mathrm{E}-06$ & $9.53674 \mathrm{E}-07$ \\
$10^{-6}$ & $8.02338 \mathrm{E}-04$ & $1.99616 \mathrm{E}-04$ & $4.99785 \mathrm{E}-05$ & $1.29044 \mathrm{E}-05$ & $3.15905 \mathrm{E}-06$ & $9.53674 \mathrm{E}-07$ \\
$10^{-8}$ & $8.02338 \mathrm{E}-04$ & $1.99616 \mathrm{E}-04$ & $4.99785 \mathrm{E}-05$ & $1.29044 \mathrm{E}-05$ & $3.15905 \mathrm{E}-06$ & $9.53674 \mathrm{E}-07$ \\
$10^{-10}$ & $8.02338 \mathrm{E}-04$ & $1.99616 \mathrm{E}-04$ & $4.99785 \mathrm{E}-05$ & $1.29044 \mathrm{E}-05$ & $3.15905 \mathrm{E}-06$ & $9.53674 \mathrm{E}-07$ \\
$10^{-15}$ & $8.02338 \mathrm{E}-04$ & $1.99616 \mathrm{E}-04$ & $4.99785 \mathrm{E}-05$ & $1.29044 \mathrm{E}-05$ & $3.15905 \mathrm{E}-06$ & $9.53674 \mathrm{E}-07$ \\
$10^{-20}$ & $8.02338 \mathrm{E}-04$ & $1.99616 \mathrm{E}-04$ & $4.99785 \mathrm{E}-05$ & $1.29044 \mathrm{E}-05$ & $3.15905 \mathrm{E}-06$ & $9.53674 \mathrm{E}-07$ \\
$10^{-30}$ & $8.02338 \mathrm{E}-04$ & $1.99616 \mathrm{E}-04$ & $4.99785 \mathrm{E}-05$ & $1.29044 \mathrm{E}-05$ & $3.15905 \mathrm{E}-06$ & $9.53674 \mathrm{E}-07$ \\
\hline
\end{tabular}

The computational results(Absolute Maximum Error)presented in Table-3, 4 for example problem-2 show that the present scheme is capable of producing uniformly convergent solution in case when $\epsilon$ tends to zero for any fixed value of the step size $h=1 / N$.

Table 3 Computational results(MAE) for various values of $\epsilon$ and $N$ for example problem-2 with $\delta=0.1 * \varepsilon$.

\begin{tabular}{|c|cccccc|}
\hline$\epsilon$ & $N=8$ & $N=16$ & $N=32$ & $N=64$ & $N=128$ & $N=256$ \\
\hline $10^{-2}$ & $3.76940 \mathrm{E}-04$ & $4.19140 \mathrm{E}-04$ & $8.56072 \mathrm{E}-04$ & $8.40575 \mathrm{E}-04$ & $5.50807 \mathrm{E}-04$ & $2.98828 \mathrm{E}-04$ \\
$10^{-4}$ & $3.56883 \mathrm{E}-04$ & $8.97944 \mathrm{E}-05$ & $2.22325 \mathrm{E}-05$ & $5.96046 \mathrm{E}-06$ & $2.92063 \mathrm{E}-06$ & $7.15256 \mathrm{E}-07$ \\
$10^{-6}$ & $3.56883 \mathrm{E}-04$ & $8.97944 \mathrm{E}-05$ & $2.22325 \mathrm{E}-05$ & $5.96046 \mathrm{E}-06$ & $2.92063 \mathrm{E}-06$ & $7.15256 \mathrm{E}-07$ \\
$10^{-8}$ & $3.56883 \mathrm{E}-04$ & $8.97944 \mathrm{E}-05$ & $2.22325 \mathrm{E}-05$ & $5.96046 \mathrm{E}-06$ & $2.92063 \mathrm{E}-06$ & $7.15256 \mathrm{E}-07$ \\
$10^{-10}$ & $3.56883 \mathrm{E}-04$ & $8.97944 \mathrm{E}-05$ & $2.22325 \mathrm{E}-05$ & $5.96046 \mathrm{E}-06$ & $2.92063 \mathrm{E}-06$ & $7.15256 \mathrm{E}-07$ \\
$10^{-15}$ & $3.56883 \mathrm{E}-04$ & $8.97944 \mathrm{E}-05$ & $2.22325 \mathrm{E}-05$ & $5.96046 \mathrm{E}-06$ & $2.92063 \mathrm{E}-06$ & $7.15256 \mathrm{E}-07$ \\
$10^{-20}$ & $3.56883 \mathrm{E}-04$ & $8.97944 \mathrm{E}-05$ & $2.22325 \mathrm{E}-05$ & $5.96046 \mathrm{E}-06$ & $2.92063 \mathrm{E}-06$ & $7.15256 \mathrm{E}-07$ \\
$10^{-30}$ & $3.56883 \mathrm{E}-04$ & $8.97944 \mathrm{E}-05$ & $2.22325 \mathrm{E}-05$ & $5.96046 \mathrm{E}-06$ & $2.92063 \mathrm{E}-06$ & $7.15256 \mathrm{E}-07$ \\
\hline
\end{tabular}

Table 4 Computational results(MAE) for various values of $\epsilon$ and $N$ for example problem-2 with $\delta=0.5 * \varepsilon$.

\begin{tabular}{|c|cccccc|}
\hline$\epsilon$ & $N=8$ & $N=16$ & $N=32$ & $N=64$ & $N=128$ & $N=256$ \\
\hline $10^{-2}$ & $3.56883 \mathrm{E}-04$ & $1.10626 \mathrm{E}-04$ & $2.57373 \mathrm{E}-04$ & $5.01603 \mathrm{E}-04$ & $4.39823 \mathrm{E}-04$ & $2.84374 \mathrm{E}-04$ \\
$10^{-4}$ & $3.56883 \mathrm{E}-04$ & $8.97944 \mathrm{E}-05$ & $2.22325 \mathrm{E}-05$ & $5.96046 \mathrm{E}-06$ & $2.92063 \mathrm{E}-06$ & $7.15256 \mathrm{E}-07$ \\
$10^{-6}$ & $3.56883 \mathrm{E}-04$ & $8.97944 \mathrm{E}-05$ & $2.22325 \mathrm{E}-05$ & $5.96046 \mathrm{E}-06$ & $2.92063 \mathrm{E}-06$ & $7.15256 \mathrm{E}-07$ \\
$10^{-8}$ & $3.56883 \mathrm{E}-04$ & $8.97944 \mathrm{E}-05$ & $2.22325 \mathrm{E}-05$ & $5.96046 \mathrm{E}-06$ & $2.92063 \mathrm{E}-06$ & $7.15256 \mathrm{E}-07$ \\
$10^{-10}$ & $3.56883 \mathrm{E}-04$ & $8.97944 \mathrm{E}-05$ & $2.22325 \mathrm{E}-05$ & $5.96046 \mathrm{E}-06$ & $2.92063 \mathrm{E}-06$ & $7.15256 \mathrm{E}-07$ \\
$10^{-15}$ & $3.56883 \mathrm{E}-04$ & $8.97944 \mathrm{E}-05$ & $2.22325 \mathrm{E}-05$ & $5.96046 \mathrm{E}-06$ & $2.92063 \mathrm{E}-06$ & $7.15256 \mathrm{E}-07$ \\
$10^{-20}$ & $3.56883 \mathrm{E}-04$ & $8.97944 \mathrm{E}-05$ & $2.22325 \mathrm{E}-05$ & $5.96046 \mathrm{E}-06$ & $2.92063 \mathrm{E}-06$ & $7.15256 \mathrm{E}-07$ \\
$10^{-30}$ & $3.56883 \mathrm{E}-04$ & $8.97944 \mathrm{E}-05$ & $2.22325 \mathrm{E}-05$ & $5.96046 \mathrm{E}-06$ & $2.92063 \mathrm{E}-06$ & $7.15256 \mathrm{E}-07$ \\
\hline
\end{tabular}

Example 3 We consider the singularly perturbed delay nonlinear problem exhibiting leftend boundary layer

$$
\varepsilon y^{\prime \prime}(x)+2 y^{\prime}(x-\delta)+\exp (y(x))=0, x \in[0,1]
$$

subject to the interval and boundary conditions

The linear form is:

$$
y(x)=0,-\delta \leq x \leq 0, y(1)=0
$$

$$
\varepsilon y^{\prime \prime}(x)+2 y^{\prime}(x-\delta)+y(x)=-1 ; x \in[0,1] \text { with } y(0)=0 \text { and } y(1)=0
$$

The exact solution is not known for this problem. The absolute maximum errors are given in Tables 5 for $\delta=0.1 * \varepsilon$.

The computational results(Absolute Maximum Error)presented in Table-5 for example problem-3 show that the present scheme is capable of producing uniformly convergent solution in case when $\epsilon$ tends to zero for any fixed value of the step size $h=1 / N$. 
Table 5 Computational results(MAE) for various values of $\epsilon$ and $N$ for example problem-3 with $\delta=0.1 * \varepsilon$.

\begin{tabular}{|c|cccccc|}
\hline$\epsilon$ & $N=8$ & $N=16$ & $N=32$ & $N=64$ & $N=128$ & $N=256$ \\
\hline $10^{-2}$ & $1.66178 \mathrm{E}-04$ & $4.02927 \mathrm{E}-05$ & $1.10924 \mathrm{E}-04$ & $3.77297 \mathrm{E}-04$ & $4.20749 \mathrm{E}-04$ & $2.83182 \mathrm{E}-04$ \\
$10^{-4}$ & $1.65641 \mathrm{E}-04$ & $4.58956 \mathrm{E}-05$ & $1.19209 \mathrm{E}-05$ & $3.45707 \mathrm{E}-06$ & $2.98023 \mathrm{E}-07$ & $2.38419 \mathrm{E}-07$ \\
$10^{-6}$ & $1.65641 \mathrm{E}-04$ & $4.58956 \mathrm{E}-05$ & $1.19209 \mathrm{E}-05$ & $3.45707 \mathrm{E}-06$ & $2.98023 \mathrm{E}-07$ & $2.38419 \mathrm{E}-07$ \\
$10^{-8}$ & $1.65641 \mathrm{E}-04$ & $4.58956 \mathrm{E}-05$ & $1.19209 \mathrm{E}-05$ & $3.45707 \mathrm{E}-06$ & $2.98023 \mathrm{E}-07$ & $2.38419 \mathrm{E}-07$ \\
$10^{-10}$ & $1.65641 \mathrm{E}-04$ & $4.58956 \mathrm{E}-05$ & $1.19209 \mathrm{E}-05$ & $3.45707 \mathrm{E}-06$ & $2.98023 \mathrm{E}-07$ & $2.38419 \mathrm{E}-07$ \\
$10^{-15}$ & $1.65641 \mathrm{E}-04$ & $4.58956 \mathrm{E}-05$ & $1.19209 \mathrm{E}-05$ & $3.45707 \mathrm{E}-06$ & $2.98023 \mathrm{E}-07$ & $2.38419 \mathrm{E}-07$ \\
$10^{-20}$ & $1.65641 \mathrm{E}-04$ & $4.58956 \mathrm{E}-05$ & $1.19209 \mathrm{E}-05$ & $3.45707 \mathrm{E}-06$ & $2.98023 \mathrm{E}-07$ & $2.38419 \mathrm{E}-07$ \\
$10^{-30}$ & $1.65641 \mathrm{E}-04$ & $4.58956 \mathrm{E}-05$ & $1.19209 \mathrm{E}-05$ & $3.45707 \mathrm{E}-06$ & $2.98023 \mathrm{E}-07$ & $2.38419 \mathrm{E}-07$ \\
\hline
\end{tabular}

Example 4 Now we consider an example of variable coefficient singularly perturbed delay differential equation exhibiting right-end boundary layer:

$$
\varepsilon y^{\prime \prime}(x)-e^{x} y^{\prime}(x-\delta)-x y(x)=0 ; x \in[0,1]
$$

subject to the boundary conditions

$$
y(0)=1 \text { and } y(1)=1
$$

The exact solution is not known for this problem. The absolute maximum errors are given in Tables 6,7 for $\delta=0.1 * \varepsilon$ and $\delta=0.5 * \varepsilon$ respectively.

The computational results(Absolute Maximum Error)presented in Table-6, 7 for example problem-4 show that the present scheme is capable of producing uniformly convergent solution in case when $\epsilon$ tends to zero for any fixed value of the step size $h=1 / N$.

Table 6 Computational results(MAE) for various values of $\epsilon$ and $N$ for example problem- 4 with $\delta=0.1 * \varepsilon$.

\begin{tabular}{|c|cccccc|}
\hline$\epsilon$ & $N=8$ & $N=16$ & $N=32$ & $N=64$ & $N=128$ & $N=256$ \\
\hline $10^{-2}$ & $3.52561 \mathrm{E}-04$ & $1.42097 \mathrm{E}-04$ & $3.61681 \mathrm{E}-04$ & $4.13740 \mathrm{E}-03$ & $2.21390 \mathrm{E}-02$ & $5.56450 \mathrm{E}-02$ \\
$10^{-4}$ & $3.51191 \mathrm{E}-04$ & $9.26852 \mathrm{E}-05$ & $2.41399 \mathrm{E}-05$ & $5.66244 \mathrm{E}-06$ & $2.20537 \mathrm{E}-06$ & $1.25170 \mathrm{E}-06$ \\
$10^{-6}$ & $3.51191 \mathrm{E}-04$ & $9.26852 \mathrm{E}-05$ & $2.41399 \mathrm{E}-05$ & $5.66244 \mathrm{E}-06$ & $2.20537 \mathrm{E}-06$ & $1.25170 \mathrm{E}-06$ \\
$10^{-8}$ & $3.51191 \mathrm{E}-04$ & $9.26852 \mathrm{E}-05$ & $2.41399 \mathrm{E}-05$ & $5.66244 \mathrm{E}-06$ & $2.20537 \mathrm{E}-06$ & $1.25170 \mathrm{E}-06$ \\
$10^{-10}$ & $3.51191 \mathrm{E}-04$ & $9.26852 \mathrm{E}-05$ & $2.41399 \mathrm{E}-05$ & $5.66244 \mathrm{E}-06$ & $2.20537 \mathrm{E}-06$ & $1.25170 \mathrm{E}-06$ \\
$10^{-15}$ & $3.51191 \mathrm{E}-04$ & $9.26852 \mathrm{E}-05$ & $2.41399 \mathrm{E}-05$ & $5.66244 \mathrm{E}-06$ & $2.20537 \mathrm{E}-06$ & $1.25170 \mathrm{E}-06$ \\
$10^{-20}$ & $3.51191 \mathrm{E}-04$ & $9.26852 \mathrm{E}-05$ & $2.41399 \mathrm{E}-05$ & $5.66244 \mathrm{E}-06$ & $2.20537 \mathrm{E}-06$ & $1.25170 \mathrm{E}-06$ \\
$10^{-30}$ & $3.51191 \mathrm{E}-04$ & $9.26852 \mathrm{E}-05$ & $2.41399 \mathrm{E}-05$ & $5.66244 \mathrm{E}-06$ & $2.20537 \mathrm{E}-06$ & $1.25170 \mathrm{E}-06$ \\
\hline
\end{tabular}

Table 7 Computational results(MAE) for various values of $\epsilon$ and $N$ for example problem- 4 with $\delta=0.5 * \varepsilon$.

\begin{tabular}{|c|cccccc|}
\hline$\epsilon$ & $N=8$ & $N=16$ & $N=32$ & $N=64$ & $N=128$ & $N=256$ \\
\hline $10^{-2}$ & $3.62873 \mathrm{E}-04$ & $2.80082 \mathrm{E}-04$ & $7.30276 \mathrm{E}-04$ & $9.68742 \mathrm{E}-03$ & $3.57798 \mathrm{E}-02$ & $7.07997 \mathrm{E}-02$ \\
$10^{-4}$ & $3.51191 \mathrm{E}-04$ & $9.26852 \mathrm{E}-05$ & $2.41399 \mathrm{E}-05$ & $5.66244 \mathrm{E}-06$ & $2.20537 \mathrm{E}-06$ & $1.43051 \mathrm{E}-06$ \\
$10^{-6}$ & $3.51191 \mathrm{E}-04$ & $9.26852 \mathrm{E}-05$ & $2.41399 \mathrm{E}-05$ & $5.66244 \mathrm{E}-06$ & $2.20537 \mathrm{E}-06$ & $1.43051 \mathrm{E}-06$ \\
$10^{-8}$ & $3.51191 \mathrm{E}-04$ & $9.26852 \mathrm{E}-05$ & $2.41399 \mathrm{E}-05$ & $5.66244 \mathrm{E}-06$ & $2.20537 \mathrm{E}-06$ & $1.43051 \mathrm{E}-06$ \\
$10^{-10}$ & $3.51191 \mathrm{E}-04$ & $9.26852 \mathrm{E}-05$ & $2.41399 \mathrm{E}-05$ & $5.66244 \mathrm{E}-06$ & $2.20537 \mathrm{E}-06$ & $1.43051 \mathrm{E}-06$ \\
$10^{-15}$ & $3.51191 \mathrm{E}-04$ & $9.26852 \mathrm{E}-05$ & $2.41399 \mathrm{E}-05$ & $5.66244 \mathrm{E}-06$ & $2.20537 \mathrm{E}-06$ & $1.43051 \mathrm{E}-06$ \\
$10^{-20}$ & $3.51191 \mathrm{E}-04$ & $9.26852 \mathrm{E}-05$ & $2.41399 \mathrm{E}-05$ & $5.66244 \mathrm{E}-06$ & $2.20537 \mathrm{E}-06$ & $1.43051 \mathrm{E}-06$ \\
$10^{-30}$ & $3.51191 \mathrm{E}-04$ & $9.26852 \mathrm{E}-05$ & $2.41399 \mathrm{E}-05$ & $5.66244 \mathrm{E}-06$ & $2.20537 \mathrm{E}-06$ & $1.43051 \mathrm{E}-06$ \\
\hline
\end{tabular}

Example 5 Finally we consider an example of constant coefficient singularly perturbed delay differential equation exhibiting right-end boundary layer:

subject to the boundary conditions

$$
\varepsilon y^{\prime \prime}(x)-y^{\prime}(x-\delta)-y(x)=0 ; x \in[0,1]
$$

$$
y(0)=1 \text { and } y(1)=-1
$$


The exact solution is not known for this problem. The absolute maximum errors are given in Tables 8, 9 for $\delta=0.1 * \varepsilon$ and $\delta=0.5 * \varepsilon$ respectively.

The computational results(Absolute Maximum Error) presented in Table-8, 9 for example problem-5 show that the present scheme is capable of producing uniformly convergent solution in case when $\epsilon$ tends to zero for any fixed value of the step size $h=1 / N$.

Table 8 Computational results(MAE) for various values of $\epsilon$ and $N$ for example problem-5 with $\delta=0.1 * \varepsilon$.

\begin{tabular}{|c|cccccc|}
\hline$\epsilon$ & $N=8$ & $N=16$ & $N=32$ & $N=64$ & $N=128$ & $N=256$ \\
\hline $10^{-2}$ & $4.27544 \mathrm{E}-04$ & $6.99192 \mathrm{E}-04$ & $1.26231 \mathrm{E}-03$ & $1.33480 \mathrm{E}-03$ & $9.66229 \mathrm{E}-04$ & $5.69962 \mathrm{E}-04$ \\
$10^{-4}$ & $3.56942 \mathrm{E}-04$ & $8.97646 \mathrm{E}-05$ & $2.23517 \mathrm{E}-05$ & $5.87106 \mathrm{E}-06$ & $1.25170 \mathrm{E}-06$ & $4.08292 \mathrm{E}-06$ \\
$10^{-6}$ & $3.56942 \mathrm{E}-04$ & $8.97646 \mathrm{E}-05$ & $2.23517 \mathrm{E}-05$ & $5.87106 \mathrm{E}-06$ & $1.25170 \mathrm{E}-06$ & $7.15256 \mathrm{E}-07$ \\
$10^{-8}$ & $3.56942 \mathrm{E}-04$ & $8.97646 \mathrm{E}-05$ & $2.23517 \mathrm{E}-05$ & $5.87106 \mathrm{E}-06$ & $1.25170 \mathrm{E}-06$ & $7.15256 \mathrm{E}-07$ \\
$10^{-10}$ & $3.56942 \mathrm{E}-04$ & $8.97646 \mathrm{E}-05$ & $2.23517 \mathrm{E}-05$ & $5.87106 \mathrm{E}-06$ & $1.25170 \mathrm{E}-06$ & $7.15256 \mathrm{E}-07$ \\
$10^{-15}$ & $3.56942 \mathrm{E}-04$ & $8.97646 \mathrm{E}-05$ & $2.23517 \mathrm{E}-05$ & $5.87106 \mathrm{E}-06$ & $1.25170 \mathrm{E}-06$ & $7.15256 \mathrm{E}-07$ \\
$10^{-20}$ & $3.56942 \mathrm{E}-04$ & $8.97646 \mathrm{E}-05$ & $2.23517 \mathrm{E}-05$ & $5.87106 \mathrm{E}-06$ & $1.25170 \mathrm{E}-06$ & $7.15256 \mathrm{E}-07$ \\
$10^{-30}$ & $3.56942 \mathrm{E}-04$ & $8.97646 \mathrm{E}-05$ & $2.23517 \mathrm{E}-05$ & $5.87106 \mathrm{E}-06$ & $1.25170 \mathrm{E}-06$ & $7.15256 \mathrm{E}-07$ \\
\hline
\end{tabular}

Table 9 Computational results(MAE) for various values of $\epsilon$ and $N$ for example problem-5 with $\delta=0.5 * \varepsilon$.

\begin{tabular}{|c|cccccc|}
\hline$\epsilon$ & $N=8$ & $N=16$ & $N=32$ & $N=64$ & $N=128$ & $N=256$ \\
\hline $10^{-2}$ & $6.74665 \mathrm{E}-04$ & $1.34805 \mathrm{E}-03$ & $1.86940 \mathrm{E}-03$ & $1.56067 \mathrm{E}-03$ & $1.04945 \mathrm{E}-03$ & $5.34110 \mathrm{E}-04$ \\
$10^{-4}$ & $3.56942 \mathrm{E}-04$ & $8.97646 \mathrm{E}-05$ & $2.23517 \mathrm{E}-05$ & $5.87106 \mathrm{E}-06$ & $1.25170 \mathrm{E}-06$ & $1.34110 \mathrm{E}-06$ \\
$10^{-6}$ & $3.56942 \mathrm{E}-04$ & $8.97646 \mathrm{E}-05$ & $2.23517 \mathrm{E}-05$ & $5.87106 \mathrm{E}-06$ & $1.25170 \mathrm{E}-06$ & $7.15256 \mathrm{E}-07$ \\
$10^{-8}$ & $3.56942 \mathrm{E}-04$ & $8.97646 \mathrm{E}-05$ & $2.23517 \mathrm{E}-05$ & $5.87106 \mathrm{E}-06$ & $1.25170 \mathrm{E}-06$ & $7.15256 \mathrm{E}-07$ \\
$10^{-10}$ & $3.56942 \mathrm{E}-04$ & $8.97646 \mathrm{E}-05$ & $2.23517 \mathrm{E}-05$ & $5.87106 \mathrm{E}-06$ & $1.25170 \mathrm{E}-06$ & $7.15256 \mathrm{E}-07$ \\
$10^{-15}$ & $3.56942 \mathrm{E}-04$ & $8.97646 \mathrm{E}-05$ & $2.23517 \mathrm{E}-05$ & $5.87106 \mathrm{E}-06$ & $1.25170 \mathrm{E}-06$ & $7.15256 \mathrm{E}-07$ \\
$10^{-20}$ & $3.56942 \mathrm{E}-04$ & $8.97646 \mathrm{E}-05$ & $2.23517 \mathrm{E}-05$ & $5.87106 \mathrm{E}-06$ & $1.25170 \mathrm{E}-06$ & $7.15256 \mathrm{E}-07$ \\
$10^{-30}$ & $3.56942 \mathrm{E}-04$ & $8.97646 \mathrm{E}-05$ & $2.23517 \mathrm{E}-05$ & $5.87106 \mathrm{E}-06$ & $1.25170 \mathrm{E}-06$ & $7.15256 \mathrm{E}-07$ \\
\hline
\end{tabular}

\section{Discussion and Conclusions}

A numerical integration method with an exponential fitting factor has been presented for solving singularly perturbed delay differential equations, whose solutions exhibit layer behavior on one end(left or right) point of the interval considered. The effect of a negative shift on the boundary layer solutions has been investigated and presented in Tables 1-9.

One can easily observed from these Tables: $1-9$ that the presented fitted scheme is capable of producing highly accurate uniformly convergent solution for any fixed value of step size $h=(1 / N)>\epsilon$, when perturbation parameter $\varepsilon$ tends to zero. The main feature of the proposed fitted scheme is that it does not depends on the very fine mess size.

\section{References}

1. Aziz, T., Khan, A.: A spline method for second-order singularly perturbed boundaryproblems, J. Comput. Appl. Math. 147 (2), 445-452 (2002).

2. Bender, C. M., Orszag, S. A. : Advanced Mathematical Methods for Scientists and Engineers, McGraw-Hill, (1978).

3. Bestehorn, M., Grigorieva, E.V.: Formation and propagation of localized states in extended systems, Annalen der Physik, 13, 423-431 (2004). 
4. Doolan, E. P., Miller, J. J. H., Schilders, W. H. A.: Uniform Numerical methods for problems with initial and boundary layers, Boole, (1980).

5. Eckhaus, W. : Matched Asymptotic Expansions and Singular Perturbations, North Holland, Amsterdam, (1973).

6. Ezzat, M.A., Othman, M.I., El-Karamany, A.M.S.: State space approach to twodimensional generalized thermo-viscoelasticity with two relaxation times, International journal of Engineering Science, 11, 1251-1274 (2002).

7. Geng, F. Z., Qian, S. P.: Improved Reproducing Kernel Method for Singularly Perturbed Differential-Difference Equations with Boundary Layer behavior, Appl. Math. Comput. 252, 58-63 (2015).

8. Holmes, M.H.: Introduction to Perturbation Methods, Springer, Berlin, (1995).

9. Joseph, D.D., Preziosi, L.: Heat waves, Reviews of modern physics, 1 (6), 41-73 (1989).

10. Joseph, D.D., Preziosi, L.: Addendum to the paper heat waves, Reviews of modern physics, 2 (62), 375-391 (1990).

11. Kevorkian, J., Cole, J. D.: Perturbation Methods in Applied Mathematics, Springer, (1981).

12. Kadalbajoo, M. K., Gupta, V.: A brief survey on numerical methods for solving singularly perturbed problems, Appl. Math. Comput. 217, 3641-3716 (2010).

13. Kadalbajoo, M. K., Patidar, K. C.: A survey of numerical techniques for solving singularly perturbed ordinary differential equations, Appl. Math. Comput. 130, 457-510 (2002).

14. Kadalbajoo, M.K., Sharma, K.K.: Numerical Analysis of Singularly Perturbed Delay Differential Equations with Layer Behavior, Applied Mathematics and Computation, 157 (1), 11-28 (2004).

15. Kadalbajoo, M.K., Ramesh, V.P.: Hybrid Method forNumerical Solution of Singularly Perturbed Delay Differential Equations, Applied Mathematics and Computation, 187 (2), 797-814 (2007).

16. Kadalbajoo, M.K., Sharma, K.K.: Numerical Method Based on Finite Difference for Boundary Value Problems for Singularly Perturbed Delay Differential Equations, Applied Mathematics and Computation, 197 (2), 692-707 (2008).

17. Kadalbajoo, M.K., Kumar, D.: Fitted Mesh B-Spline Collocation Method for Singularly Perturbed DifferentialDifference Equations with Small Delay, Applied Mathematics and Computation, 204 (1), 90-98 (2008).

18. Kadalbajoo, M.K., Kumar, D.: Computational Method for Singularly Perturbed Nonlinear Differential-Difference Equations with Small Shift, Applied Mathematical Modeling, 34 (9), 2584-2596 (2010).

19. Kadalbajoo, M. K., Sharma, K. K.: Numerical treatment of boundary value problems for second-order singularly perturbed delay differential equations, Comput. Appl. Math. 24, 151-172 (2005).

20. Kellogg, R.B., Tsan, A.: Analysis of some difference approximations for a singular perturbation problem without turning points, Math. Comp. 32, 1025-1039 (1978).

21. Kuang, Y. : Delay Differential Equations with Application in Population Dynamics, Academic Press, (1993).

22. Lange, C.G., Miura, R.M.: Singular perturbation analysis of boundary-value problems for differential-difference equations. II. Rapid oscillations and resonances, SIAM J. Appl. Math. 45 (1), 687-707 (1985).

23. Lange, C.G., Miura, R.M.: Perturbation analysis of boundary-value problems for differential difference equations. III. Turning point problems, SIAM J. Appl. Math. 45, 708-734 (1985).

24. Lange, C.G., Miura, R.M.: Singular perturbation analysis of boundary-value problems for differential-difference equations. V. Small shifts with layer behaviour, SIAM J. Appl. Math. 54, 249-272 (1994). 
25. Lange, C.G., Miura, R.M.: Singular perturbation analysis of boundary-value problems for differential-difference equations. VI. Small shifts with rapid oscillations, SIAM J. Appl. Math. 54, 273-283 (1994).

26. Lange, C.G., Miura, R.M.: Singular perturbation analysis of boundary-value problems for differential-difference equations, SIAM J. Appl. Math. 42, 502-531 (1982).

27. Liu, Q., Wang, X., Kee, D. De: Mass transport through swelling membranes, International journal of Engineering Science, 43, 1464-1470 (2005).

28. Mackey, M.C., Glass, L.: Oscillations and chaos in physiological control systems, Science, 197, 287-289 (1977).

29. Miller, J.J.H.: Singular Perturbation Problems in Chemical Physics Analytic and Computational Methods, John Wiley and Sons, (1997).

30. Miller, J.J.H., O'Riordan, E., Shishkin, G.: Fitted Numerical Methods for singularly perturbed problems: Error estimates in the maximum norm for linear problems in one and two dimension, World Scientific Publication, Singapore, (1996).

31. Nayfeh, A. H.: Perturbation Methods, Wiley, (1979).

32. O'Malley, R. E.: Introduction to Singular Perturbations, Academic Press, (1974).

33. Patidar1, K. C., Sharma, K. K.: $\varepsilon$-Uniformly convergent non-standard finite difference methods for singularly perturbed differential difference equations with small delay, Applied Mathematics and Computation, 175, 864-890 (2006).

34. Reddy, Y. N.: Numerical Treatment of Singularly Perturbed Two Point Boundary Value Problems, Ph.D. thesis, IIT, Kanpur, India, (1986).

35. Ramos, J.I.: Exponential methods for singularly perturbed ordinary differentialdifference equations, Applied Mathematics and Computation, 182, 1528-1541 (2006). 